\title{
Analysis of Image Quality Enhancement Using Colour Depth Histogram and Luminance Contrast Masking
}

\author{
Nalinee Dhuware, Bahrti Chaurasia and Yashwant Kurmi
}

\begin{abstract}
Image enhancement is essentially required for image processing. Many enhancement processes proposed based on different requirements. An analysis of image quality via colour with depth histograms and luminance with contrast masking is present in this paper. The contrast measurement can be modified for any multiresolution image enhancement framework. Experimental results satisfy the proposed algorithm ability to achieve desired improvements. Poisson noise cancellation by improving threshold values for wavelet approximation. Further, the contrast enhancement method also considers here for better improvements and reconstruction. The different combination of deconvolution process and also proper filtering methods of each elemental image provide the better result. Image quality enhancement is possible by color improvement with depth histograms and luminance with contrast masking properly.
\end{abstract}

\section{Keywords}

Contrast masking;

Multi-scale transforms;

Histogram modification;

Histogram partitioning.

\section{INTRODUCTION}

There are various methods of enhancing the image quality by the researchers. Some of these may be discussed here to produce digital images with all related colour and contrast details[1]. Image enhancement algorithms may classify as indirect or direct enhancement methodology[2]. Indirect enhancement algorithms improve images without contrast measuring of that image[3-5]. Histogram equalization (HE) and variants methods, pixels renovations, contrast stretching scheme etc. are indirect techniques[6]. There are quality enhancement for the visual display and technologically compatibilities[7]. Generally indirect algorithms for enhancement are used adaptive one-to-one mapping process. These methods adjust the intensity of the selected pixels[8]. Generally these approaches directly not quantify image contrast; as a result overenhancement may be possible. On the other hand, direct enhancement procedures developed mimicking the human visual system (HVS)[9]. This paper also analyse the histogram- image enhancement, wavelet transform image modification and depth of field based method. Further, the contrast enhancement method also considers here for better improvements and reconstruction.

The paper is organized in the following manner. In Setion II, we present an HVS-based multi-scale image enhancement method and colour and depth histograms based image enhancement method. Section III explains the result analysis and conclusions are presented in Section IV.

\section{Multi-Scale Image EnHANCEMENT}

Image quality enhancement using colour depth histogram is possible by HVS-based direct process[10]. The multi-scale division have a better impact on image enhancement. The algorithm is best fitted with matrix formula and easily processed. Multi-scale contrasts are calculated depending on the choice of the interest. One to one mapping in the transform domains are interpreted a new class of human visual system inspired multi-scale transformation. A direct process[11-14] of adjusting the global brightness is also essential for this purpose. Generally contrast mapping a difficult process due to its non-linear relationship[15]. Multistage transformation using different scale make feasible to achieve desire contrast enhancement.

\section{(A) Colour and Depth Histograms based image Enhancement}

The proposed method is developed depending on the histogram analysis. Colour and depths histograms analysis provide a good estimation regarding the subjected image. Desire enhancement of the selected image is performed by selecting pixels considering all information. For simple calculation the colour space of the HSI or colour image transform to the RGB space to calculate the HSI or Hue-Saturation-Intensity model. Histogram is modifying deepening on intensity parameter. The desire enhancement level determine by multi-scale division and adaptive one-to-one mapping. The enhance image is acquire by re-transforming it. The block diagram of the proposed HVS-based multiscale image enhancement is shown in Fig 1. 


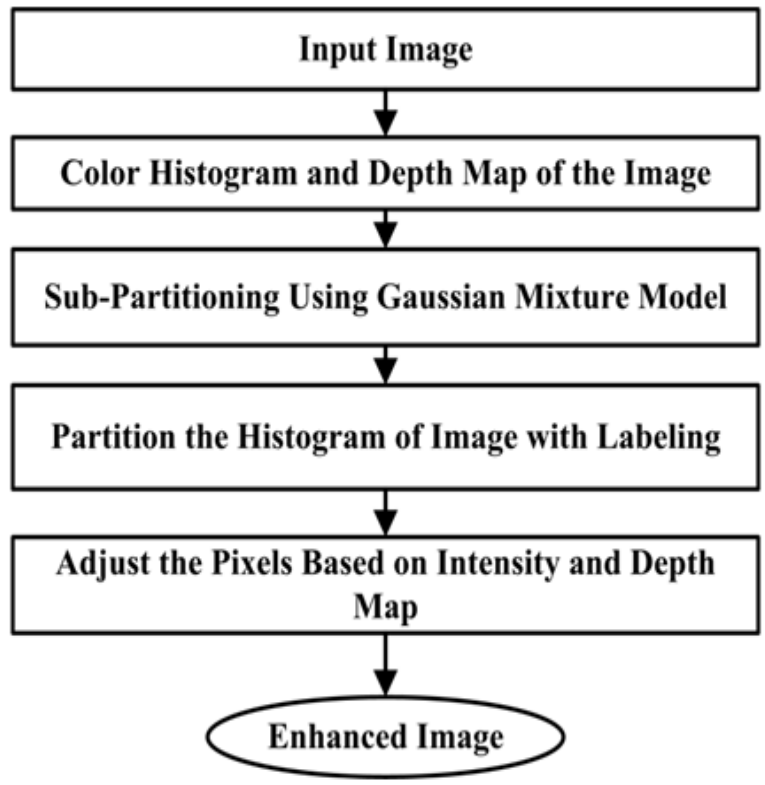

Fig 1. Flowchart of the image enhancement techniques

The whole image processing is divided into two part (i) analysis and (ii) synthesis. Schematic view of the image enhancement techniques is shown in Fig 2. Input image

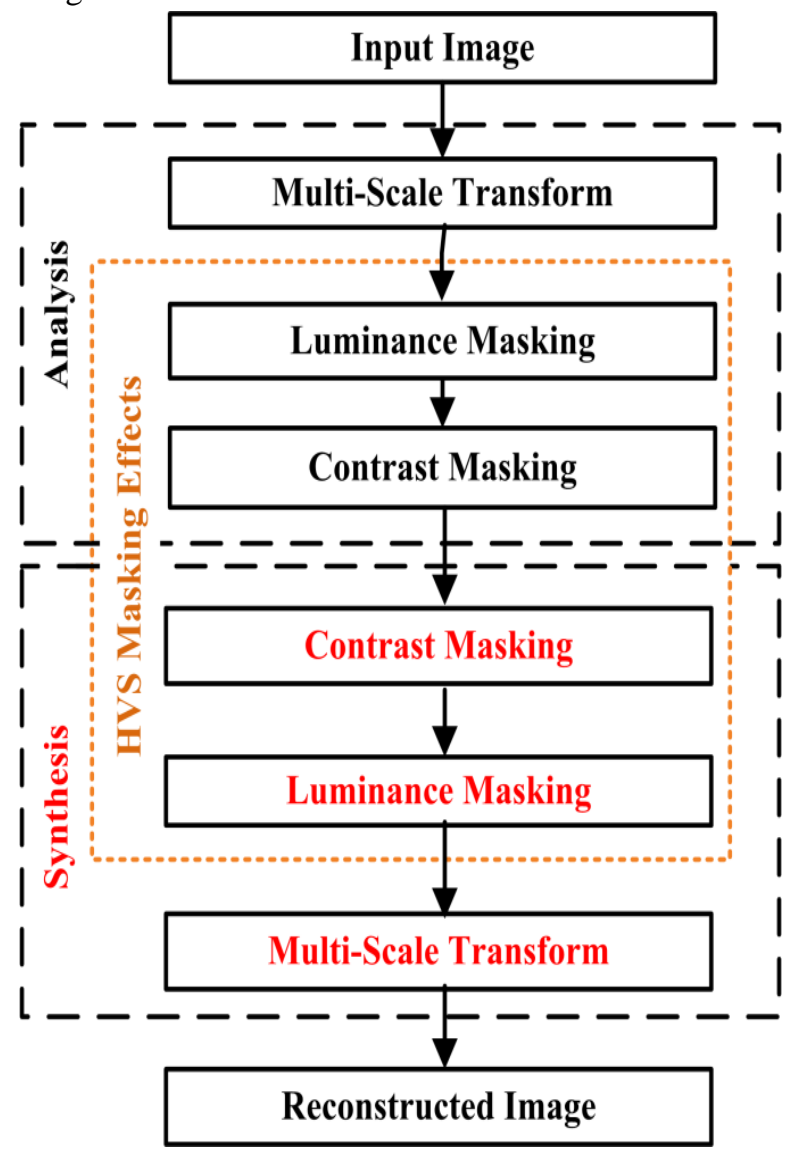

Fig 2. (Systematic view of the image enhancement techniques. Contrast masking and luminance masking perform two times during analysis and synthesis. basically transfer into multiscale and the mapping values are store in an array for future reconstruction.

To get the depth of proper contrast luminance masked. Contrast masking here perform two times during analysis and synthesis. During synthesis based on the desired value of the contrast and luminance are adjusted. Proper transfer coefficients are essential to reconstruct the image to avoid mismatch.

The HDMR is also commonly used in ANalysis Of VAriance (ANOVA) studies in statistics. The terms in the HDMR expansion are then used to calculate the sensitivity indices which show the individual and cooperative effects of model inputs on the model output. The approximate solution is provided by optimizing the first order HDMR functions. The Legendre polynomials HDMR terms to be able to reduce the sampling effort.

The HDMR is expressed by $f\left(x_{1}, \ldots, x_{n}\right)$ in $\mathrm{n}$ dimentional

$\mathrm{f}(\mathrm{x})=\mathrm{f} 0+\mathrm{n}$

$\mathrm{i}=1 \mathrm{fi}+\mathrm{n} \mathrm{i,j=1} \mathrm{i}<\mathrm{j}$ fij $+\cdots+\mathrm{f} 1 \ldots \mathrm{n}$.

By imposing the following minimizing condition of $\mathrm{dx}$ fi1...ik $(x i 1, \ldots$, xik $)=0$

if $\mathrm{j} \in\{\mathrm{i} 1, \ldots, \mathrm{ik}\}$ which allows to be determined step by step approximation process.

\section{ANALYSIS}

The experimental results obtained using the HVSinspire enhancement algorithm and color and depth histograms based image Enhancement algorithms are proposed here.

The proposed enhancement scheme is the composition use the masking of luminance as well as contrast. The extension of the non-linear is one to one mapping are based on multi-scale contrast coefficients. The overall brightness adjustment is essential.

The HVS-based transforms were derived, which are the combination of the existing resolution transformation along with HVS. The new image enhancement algorithm. the non-linear modified contrast mappings enhance HVS coefficients and contrast definitions.

Approximation the desired mapping curve of the contrast modification for each sub block, the hole image contrast may enhanced without over-enhancing the other undesired image sub block.

Figure 2, result are shown for different steps. Desire enhancement of the selected image is performed by selecting pixels considering all information. 


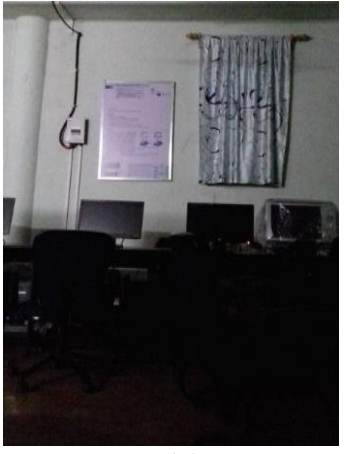

(a)

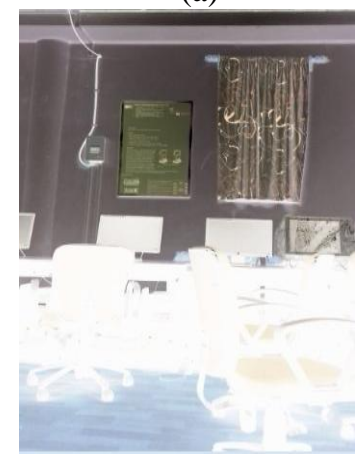

(c)

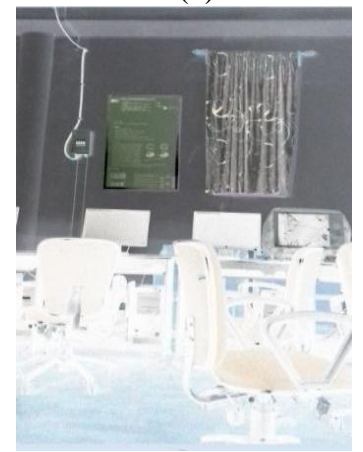

(e)

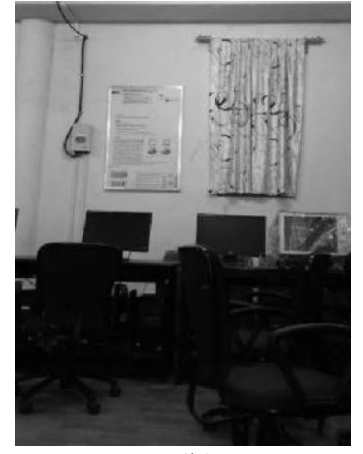

(b)

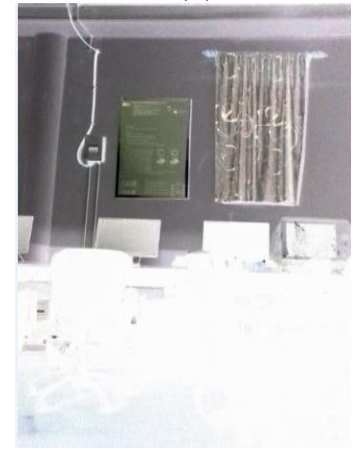

(d)

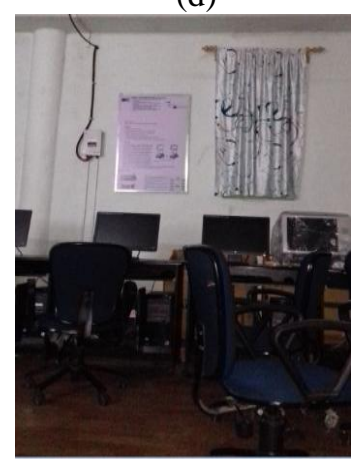

(f)
Fig 2. Output of the image enhancement process (a) original images, (b) gray scale images (c) luminance masking (d)contrast masking (e) multi-scale enhancement (f)Final enhance image.

\section{CONCLUSION}

The image enhancement algorithms using colour depth histogram and luminance contrast masking is one of the best approaches for image enhancement. The desire enhancement level determine by multi-scale division and adaptive one-to-one mapping. The result of the colour and depth histograms based image enhancement method shows improved in term of parameter and visual system performance. One to one mapping in the transform domains are interpreted a new class of human visual system inspired multi-scale transformation. A direct process of the adjusting the global brightness reduce the processing time. Experimental results satisfy the proposed algorithm ability to achieve desired improvements.

\section{REFERENCES}

[1] Jung, S. W. (2014). Image contrast enhancement using color and depth histograms. IEEE Signal Processing Letters, 21(4), 382-385.

[2] Navarro Fructuoso, H., Saavedra Tortosa, G., Martínez Corral, M., Sjöström, M., \& Olsson, R. (2014). Depthof-field enhancement in integral imaging by selective depth-deconvolution. Journal Of Display Technology, 2014, vol. 10, p. 182-188.

[3] Panetta, K. A., Wharton, E. J., \& Agaian, S. S. (2008). Human visual system-based image enhancement and logarithmic contrast measure. IEEE Transactions on Systems, Man, and Cybernetics, Part B (Cybernetics), 38(1), 174-188.

[4] Bhanu, B., Peng, J., Huang, T., \& Draper, B. (2005). Introduction to the special issue on learning in computer vision and pattern recognition. IEEE Transactions on Systems, Man, and Cybernetics, Part B (Cybernetics), 35(3), 391-396.

[5] Nercessian, S. C., Panetta, K. A., \& Agaian, S. S. (2013). Non-linear direct multi-scale image enhancement based on the luminance and contrast masking characteristics of the human visual system. IEEE Transactions on image processing, 22(9), 3549-3561.

[6] Sharma, D., Kurmi, Y., \& Chaurasia, V. (2014). Formation of Super-Resolution Image: A Review. Int. Jour. of Emerging Tech. and Adv. Engg, 4(4), 218-221.

[7] Kurmi, Y., \& Chaurasia, V. (2015). An Image Fusion Approach based on Adaptive Fuzzy Logic Model with Local Level Processing. International Journal of Computer Applications, 124(1).

[8] Tiwari, S., Chauhan, K., \& Kurmi, Y. (2015). Shadow detection and compensation in aerial images using MATLAB. International Journal of Computer Applications, 119(20).

[9] Kurmi, Y., \& Chaurasia, V. (2014). Performance of Haze Removal Filter for Hazy and Noisy Images. Int. Jour. of Sci. Engg. and Tech, 3(4), 437-439.

[10] Kumar, A., \& Chourasia, B. (2017). Image Dehazing (Defogging) by using Depth Estimation and Fusion with Guided Filter. International Journal of Computer Applications, 158(8).

[11] Kumar, A., \& Chourasia, B. (2017). Image Dehazing (Defogging) by using Depth Estimation and Fusion with Guided Filter. International Journal of Computer Applications, 158(8).

[12] Agaian, S. S., Silver, B., \& Panetta, K. A. (2007). Transform coefficient histogram-based image enhancement algorithms using contrast entropy. IEEE transactions on image processing, 16(3), 741-758.

[13] Xia, J., Panetta, K., \& Agaian, S. (2011, February). Color image enhancement algorithm based on logarithmic transform coefficient histogram. In IS\&T/SPIE Electronic Imaging (pp. 78700Y78700Y). International Society for Optics and Photonics.

[14] Wharton, E., Panetta, K., \& Agaian, S. (2007, February). Adaptive multi-histogram equalization using human vision thresholding. In Electronic Imaging 2007 (pp. 
64970G-64970G). International Society for Optics and Photonics.

[15] Wharton, E., Panetta, K., \& Againan, S. (2007, April). Human visual system based multi-histogram equalization for non-uniform illumination and shoadow correction. In Acoustics, Speech and Signal Processing, 2007. ICASSP 2007. IEEE International Conference on (Vol. 1, pp. I-729). IEEE.

\section{Nalinee Dhuware}

Dept. of ECE, Scope college of Engineering, Bhopal, India setu17687@gmail.com

\section{Bharti Chaurasia}

Dept. of ECE, Scope college of Engineering, Bhopal, India

bharti.chourasia27@gmail.com

\section{Yashwant Kurmi}

Dept. of ECE, Maulana Azad National Institute of Technology Bhopal, India yashwantkurmi18@gmail.com

\section{Editor-in-Chief Sahadev Roy}

